\title{
Prematurity, immune function and infant feeding practices
}

\author{
Shelagh M. Hampton \\ Endocrinology and Metabolism Group, School of Biological Sciences, University of Surrey, Guildford GU2 5XH, UK
}

\begin{abstract}
Recently, there has been much interest in the literature in the role of early nutrition and the health of the individual in adulthood. A majority of infants in the UK are born full term, while preterm infants account for 4-6\% of the total births. Milk feeding practices are divided into three groups: breast, combination (breast-fed with formula as 'top-up') and bottle (formula). In studies conducted by our group and other researchers immune function in full-term and preterm infants has been assessed by monitoring total and specific immunoglobulin $\mathrm{E}$ and specific immunoglobulin $G$ levels. Dietary modification by the pregnant mother with a history of allergy in the family has been shown to have a positive effect with respect to allergy outcome and prevention of atopic disease in the infant. However, this dietary modification has to occur before week 22 of pregnancy and continue until the end of lactation to achieve a beneficial outcome to the infant. The stress of mothers restricting their diets may be disadvantageous to the fetus, and therefore any gain due to the dietary restriction may be lost. Researchers have shown that the early introduction of complementary foods and the greater diversity of these foods appeared to result in an increase in the incidence of atopic disease in the infant. In conclusion, in order to reduce the risk in their babies, mothers with a family history of atopic disease should breast-feed for more than 15 weeks and introduce solid foods after 4 months, limiting the variety until at least 6 months.
\end{abstract}

Infant feeding practices: Immune function: Atopic disease

The incidence of allergy is rising. The present review will address the important issues of milk feeding practices and introduction of complementary foods with respect to allergy outcome and prevention of atopic disease.

A majority of babies in the UK are born full term (37-42 weeks), and preterm babies (those born before 37 weeks) account for 4-6\% of the total births, with this number set to rise with the development of new medical techniques. The major causes of preterm births are medical, such as spontaneous ruptured membranes and hypertension, and social, such as smoking and alcoholism. The majority of babies are breast-fed, although in the preterm infant combination feeding appears to be the most common milk feeding regimen. This involves breast-feeding, with 'top-up' formula feeds being given in the hospitals or at home when the infant is nutritionally and physiologically ready for the introduction of complementary foods.

Intestinal permeability occurs during the neonatal period. More food antigens pass across the gut of preterm infants than full-term infants (Roberton et al. 1982). Early administration of antigen delays, while breast-feeding speeds up, the rate of gut closure (Arvola et al. 1992). Immune function in the neonate has been assessed by monitoring total and specific immunoglobulin E (Hattevig et al. 1993; Sigurs et al. 1994) and specific immunoglobulin G levels (FälthMagnusson et al. 1988; Lovegrove et al. 1994). Recently, eosinophilic cationic protein and peripheral-blood mononuclear responses have been monitored in babies, with some interesting results. Jones et al. (1996) have demonstrated, by monitoring proliferation responses, that primary sensitization to an allergen occurs as early as week 22 of gestation. Eosinophilic cationic protein levels have been shown to be raised in patients with atopic dermatitis. Motolese et al. (1998) have demonstrated that eosinophilic cationic protein may be a suitable variable for monitoring the severity of atopic dermatitis in patients.

\section{Modification of diet during pregnancy and lactation}

A number of studies have investigated the role of dietary modification and allergy outcome in infants from atopic families.

Cant et al. (1986) studied the effect of eliminating milk and eggs from the diets of mothers who were breast-feeding 
their infants. The infants had all presented at the hospital with eczema, and this was monitored for extent and severity throughout the study. The mothers were placed on a 12week diet divided into three periods of 4 weeks. This study was a double-blind crossover design, in which the mothers eliminated cow's milk, eggs, chocolate, wheat, nuts, fish, beef, citrus fruits, colouring and preservative from their normal diet. They were placed in random order for 4 weeks on each of two substitute diets (a) soyabean and (b) cow's milk and egg powder, and returned to their normal diet for 4 weeks at the end of the study. The condition improved in six of the children studied, and Cant et al. (1986) suggested that there was a subgroup of children whose condition was improved when the mothers were on a restricted diet.

Sigurs et al. (1992) extended the studies of Cant et al. (1986) by placing mothers on an elimination diet for the first 3 months of breast-feeding. This observational study recruited 115 mothers with a family history of atopic disease and divided them into two groups. One group of mothers avoided cow's milk, fish and eggs during the first 3 months of lactation, and the other group kept to their normal diet. The infants were assessed at 3,6 and 48 months, and a significantly higher incidence of eczema was observed in the children whose mothers did not follow the elimination diet during lactation (Fig. 1).

A group of Swedish researchers (Fälth-Magnusson \& Kjellman, 1992) investigated 209 mothers with a history of atopic disease and divided them into two groups. One group of mothers eliminated cow's milk and eggs from their diet from week 28 of pregnancy until delivery, and the other group adhered to their normal diet. There was no reduction in atopic disease in infants when mothers were placed on the elimination diets. However, primary sensitization of the baby has been shown to occur as early as week 22 of pregnancy, which may explain the lack of reduction in atopic disease in the infants whose mothers were in the elimination group.

An observational study together with monitoring of immunological markers in three groups of mothers was carried out by Lovegrove et al. (1994). One group of mothers with a family history of atopic disease and a second

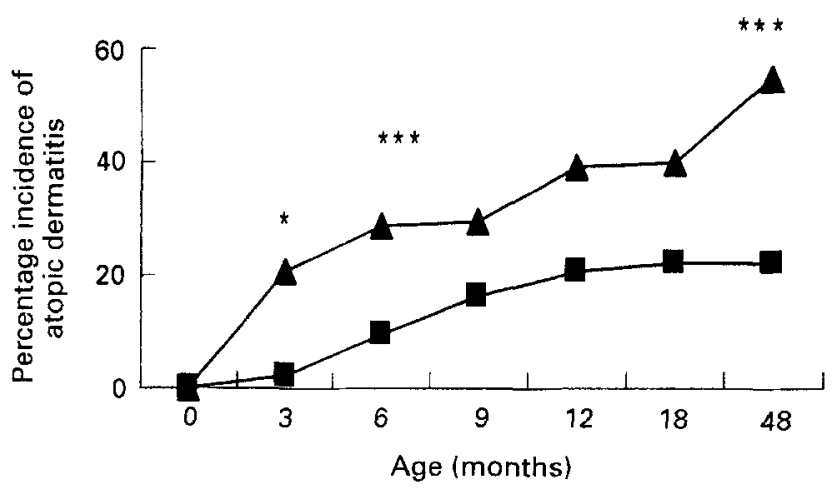

Fig. 1. Cumulative incidence of atopic dermatitis in 4-year-old children. ( $\square$ ), Mothers on dietary restriction (avoided cow's milk, fish and eggs during first 3 months of lactation); $(\mathbf{\Delta})$, mothers on normal diet. Mean values were significantly different from those for mothers on normal diet: ${ }^{\star} P<0.05,{ }^{\star \star \star} P<0.001$. (Adapted from Sigurs et al. 1992.)
Table 1. Allergy incidence $(A)$ and severity $(S)$ in infants bom to mothers with a family history of atopic disease, mothers with no history of atopic disease, and atopic mothers placed on an elimination diet removing cow's milk from the diet from week 36 of pregnancy (Adapted from Lovegrove et al. 1994)

\begin{tabular}{|c|c|c|c|}
\hline $\begin{array}{l}\text { Study group ... } \\
n \ldots\end{array}$ & $\begin{array}{c}\text { Atopic } \\
14\end{array}$ & $\begin{array}{c}\text { Non-atopic } \\
13\end{array}$ & $\begin{array}{c}\text { Atopic diet } \\
12\end{array}$ \\
\hline \multicolumn{4}{|l|}{12 months } \\
\hline Alt & 8 & $2^{* * *}$ & 5 \\
\hline$S \ddagger$ & 2 & 1 & 2 \\
\hline \multicolumn{4}{|l|}{18 months } \\
\hline Alt & 7 & $2^{* *}$ & $4^{*}$ \\
\hline $\mathrm{S} \ddagger$ & 2 & 1 & 1 \\
\hline
\end{tabular}

Mean values were significantly different from those for the atopic group:

${ }^{*} P<0.04,{ }^{* *} P<0.02,{ }^{* * *} P<0.008$.

$\dagger$ Occurrence of clinically-diagnosed atopic eczema.

$\mp$ Scored on a scale $1-3$, where 3 is most severe form.

group of mothers with no history of atopic disease in the family were recruited. The atopic group was further divided into two subgroups, one of which was placed on an elimination diet removing cow's milk from the diet from week 36 of pregnancy and throughout lactation, while the other group maintained their normal diet. The children with a family history of atopic disease had a significantly reduced incidence of developing allergies when mothers were placed on a cow's-milk-free diet (Table 1).

To be of any use, elimination diets must begin before week 22 of pregnancy, since Jones et al. (1996) showed that primary sensitization occurs as early as the 2 nd trimester. In a family with a history of atopic disease, placing the mother on an elimination diet together with breast-feeding of her infant, may have some benefit, but the stress to the mother of such a diet may outweigh the advantages.

\section{Introduction of complementary foods}

Complementary food is the term used for any food, whether manufactured or locally prepared, which is suitable as a complement to breast milk or to infant formulas when either becomes insufficient to satisfy the nutritional requirements of the infant. Such foods are commonly referred to as weaning foods.

The government guidelines on the introduction of complementary foods state that babies should be breast-fed for the first 4 months of life, with solid foods being introduced thereafter, and a mixed diet should be offered by the age of 6 months (Department of Health, 1994).

Studies investigating the introduction of complementary food have monitored the types of foods consumed and when they were introduced to the infant, and the allergic outcome in the infants.

Kajosaan \& Saarinen (1983) investigated 135 children from atopic families. They divided the infants into two groups; one group were exclusively breast-fed for 6 months, and the other group started consuming complementary foods from the age of 3 months. These complementary foods were cooked potatoes, carrots, berries, meat and cereals. The incidences of atopic eczema and food allergy at 1 year of age were monitored and the findings showed that 


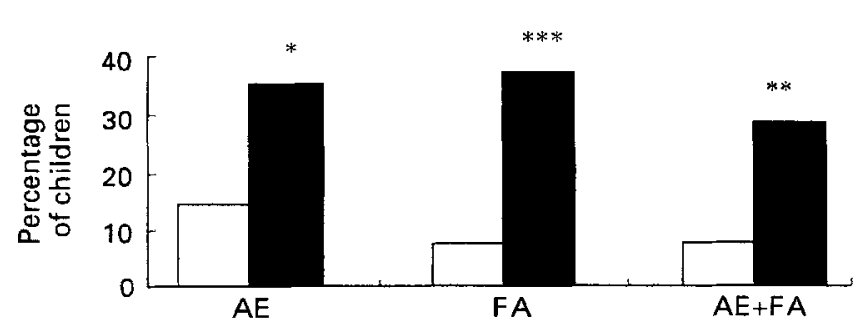

Fig. 2. Atopic eczema (AE) and food allergy (FA) at 1 year of age. $(\square)$, Infants exclusively breast-fed for 6 months; $(\square)$, infants consumed complementary food from 3 months of age. Mean values were significantly different from those for the breast-fed group: ${ }^{\star} P<0.05$, ${ }^{* \star} P<0.01,{ }^{\star \star \star} P<0.001$. (Adapted from Kajosaari \& Saarinen, 1983.)

the incidences of both disorders were significantly raised in the children who received solid foods after 3 months but before 6 months of age (Fig. 2). They concluded that children from atopic families had a reduced risk if they were exclusively breast-fed and excluded solid foods for 6 months.

Researchers in New Zealand (Fergusson et al. 1990) carried out an observational study in a group of 1210 children. They assessed the children at 4 months and annually for 10 years. They investigated the risk of developing chronic or recurrent eczema and its relationship with the early introduction of solid foods. They demonstrated that children exposed initially to four or more types of solid foods had a 2.35 times greater risk of developing eczema than children not exposed to solid feeding (Table 2). Fergusson et al. (1990) stated that it was the diet diversity and not a particular food that increased the risk of eczema.

A prospective observational study investigating 671 children in Scotland (Forsyth et al. 1993) monitored infants from 2 weeks of age, then at regular intervals up to 24 months. They divided the children into three groups, depending on when solid foods were introduced: at less than 8 weeks, between 8 and 12 weeks, at greater than 12 weeks. When solid foods were introduced at an early age there
Table 2. Risk of recurrent or chronic eczema in children in relation to early introduction of solid food (Adapted from Fergusson et al. 1990)

\begin{tabular}{lcc}
\hline $\begin{array}{l}\text { No. of solid foods } \\
(0-4 \text { months of age })\end{array}$ & No. of children & Risk of eczema \\
\hline 0 & 331 & $5 \cdot 4$ \\
$1-3$ & 755 & $7 \cdot 5^{\star}$ \\
$4+$ & 124 & $12 \cdot 7^{\star}$ \\
\hline
\end{tabular}

The risk was significantly different from that for children receiving no solid foods: ${ }^{*} P<0.05$

were significant increases in the incidences of respiratory illness at the age of 14-26 weeks and persistent cough at 14 39 weeks. The incidence of atopic eczema was increased at 53-104 weeks in the group that received solids at between 8 and 12 weeks.

A follow-up study of this same cohort for 7 years (Wilson et al. 1998) investigated different feeding patterns. They studied those children who were breast-fed exclusively for 15 weeks, and those who were breast-fed but also received solid food before 15 weeks. They showed a significant rise in the occurrence of wheeze in those children receiving solids before 15 weeks. They concluded that in order to reduce the risk of developing allergies breast-feeding should continue up to 15 weeks and no solid foods should be introduced before 15 weeks of age.

A study investigating the weaning practices and incidence of atopic disease in the preterm baby is being carried out currently by our group at the University of Surrey. This is an observational study, with immunological markers being monitored in the breast milk, and serum samples from the cord and the infant. Physical examination of the infants is being carried out at 8 and 12 months. Age of weaning from birth has been studied in 140 infants and shown to be biphasic, with peaks at 12 and 16 weeks (Fig. 3). The incidence of atopic disease at 8 and 12 months in seventy babies who have completed the project has been investigated. Dividing the infants into early- and late-weaning

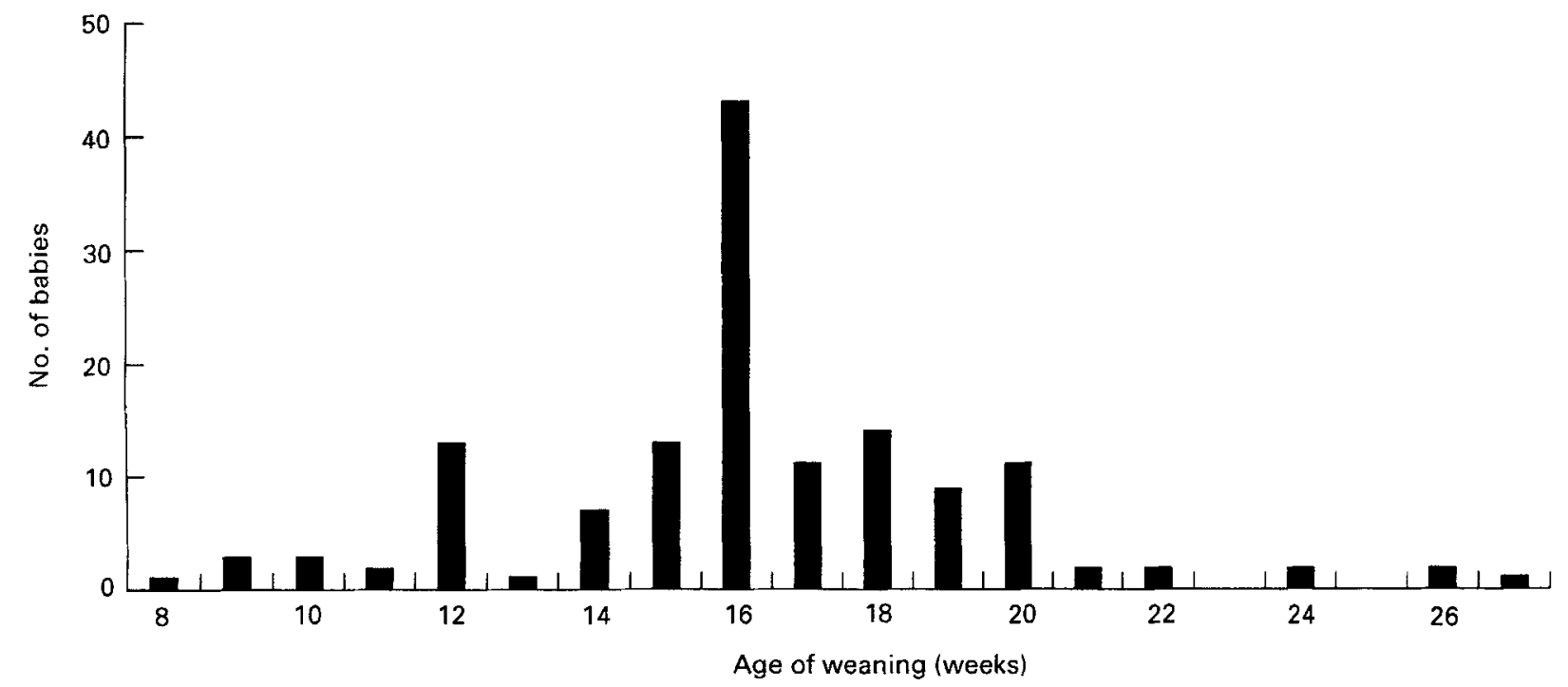

Fig. 3. Age from birth at which weaning starts in preterm infants ( $S$ Hampton, F Norris, G Williams, M Larkin and J Morgan, unpublished results). 
groups has shown that the pattern of allergies altered, depending on the age of the infants when the physical examination was carried out. Our preliminary results have shown that at 8 months babies who were weaned at less than 15 weeks had a greater risk of developing atopic disease compared with infants who were weaned after 15 weeks. However, when the same infants were re-examined at 12 months both early- and late-weaning infants had approximately the same risk of developing atopic disease. A high incidence of atopic eczema was observed.

In conclusion, in order to reduce the risk of allergy development infants should be exclusively breast-fed for more than 15 weeks, and solid foods should be introduced at 15 weeks or later. In the preterm infant this could be a late as 6 months postnatal age, although we await further information to confirm this observation. When solid foods are introduced, a small selection should be introduced up to 1 year.

What of the future? The use of hypoallergenic formulas in the prevention of atopic disease requires further investigation, particularly in the preterm infant. Further studies should investigate the introduction of complementary foods and finally studies at the molecular level are required to establish the inheritance link.

\section{Acknowledgements}

I would like to thank the Ministry of Agriculture, Fisheries and Food for its financial support and Dr Jane Morgan and Dr Fiona Norris for their valuable contributions when preparing this manuscript. I would also like to thank research midwives Chris Williams and Maggie Larkin for their support in recruiting preterm infants, and for all their efforts on the preterm baby project.

\section{References}

Arvola T, Rantala I, Marttinen A \& Isolaurii E (1992) Early dietary antigens delay the development of gut mucosal barrier in preweaning rats. Pediatric Research 32, 301-305.

Cant AJ, Bailes JA, Marsden RA \& Hewitt D (1986) Effect of maternal dietary exclusion on breast fed infants with eczema: two controlled studies. British Medical Journal 293, 231-233.

Department of Health (1994) Weaning and the Weaning Diet. Report of the Working Group on the Weaning Diet of the Committee on Medical Aspects of Food Policy. London: H. M. Stationery Office.
Fälth-Magnusson K \& Kjellman N-IM (1992) Allergy prevention by maternal elimination diet during late pregnancy: A 5-year follow-up of a randomised study. Journal of Allergy and Clinical Immunology 89, 709-713.

Fälth-Magnusson K, Kjellman N-IM \& Magnusson K-E (1988) Antibodies IgG, IgA, and IgM to food antigens during the first 18 months of life in relation to feeding and development of atopic disease. Joumal of Allergy and Clinical Immunology 81, 743749.

Fergusson DM, Horwood J \& Shannon FT (1990) Early solid feeding and recurrent childhood eczema: A longitudinal study. Pediatrics 86, 541-546.

Forsyth JS, Ogston SA, Clark A, du V Florey C \& Howie PW (1993) Relation between early introduction of solid food to infants and their weight and illnesses during the first two years of life. British Medical Journal 306, 1572-1576.

Hattevig G, Kjellman B \& Bjorksten B (1993) Appearance of IgE antibodies to ingested and inhaled allergens during the first 12 years of life in atopic and non-atopic children. Pediatric Allergy Immunology 4, 182-186.

Jones AC, Miles EA, Warner JO, Colwell BM, Bryant TN \& Warner JA (1996) Fetal peripheral blood mononuclear cell proliferative responses to mitogenic and allergenic stimuli during gestation. Pediatric Allergy Immunology 7, 109-116.

Kajosaari M \& Saarinen U (1983) Prophylaxis of atopic disease by six months total solid food elimination. Acta Paediatrica Scandinavica 72, 411-414.

Lovegrove JA, Hampton SM \& Morgan JB (1994) The immunological and long term atopic outcome of infants born to women following a milk-free diet during pregnancy and lactation: a pilot study. British Journal of Nutrition 71, 223-238.

Motolese A, Scacchetti AT \& Scarabello A (1998) Serum eosinophilic cationic protein affected by atopic dermatitis. Journal of Allergy and Clinical Immunology 101, 811 .

Roberton DM, Paganelli R \& Dinwiddle R (1982) Milk antigen absorption in the preterm and term neonate. Archives of Disease in Childhood 18, 1252-1257.

Sigurs N, Hattevig G \& Kjellman B (1992) Maternal avoidance of eggs, cow's milk and fish during lactation: Effect on allergic manifestations, skin-prick tests, and specific IgE antibodies in children at age 4 years. Pediatrics 89, 735-739.

Sigurs N, Hattevig G, Kjellman B, Kjellman N-IM, Nilsson L \& Björkstén B (1994) Appearance of atopic disease in relation to serum IgE antibodies in children followed up from birth for 4 to 15 years. Journal of Allergy and Clinical Immunology 94, 757-763.

Wilson AC, Forsyth SC, Greene SA, Irvine L, Hagau C \& Howie PW (1998) Relation of infant diet to childhood health: seven year follow up of children in Dundee infant feeding study. British Medical Journal 316, 21-25. 